\title{
Latest results of the ANTARES neutrino telescope
}

\author{
Juan-de-Dios Zornoza1a, on behalf of the ANTARES Collaboration \\ ${ }^{1}$ Instituto de Física Corpuscular, IFIC (UV-CSIC), c/ Catedrático José Beltrán 2, 46980 Paterna, Valencia (Spain)
}

\begin{abstract}
Neutrino astronomy is in an exciting moment. The discovery of a cosmic flux of high energy neutrinos by IceCube heralds a new era in which neutrinos have finally joined the multi-messenger study of the Universe. This new important window complements more "traditional" probes (as cosmic rays or photons), given the particular combination of characteristics of neutrinos (neutral, stable and weakly interacting). The ANTARES detector, built in the Mediterranean Sea, has succeeded in two key points. First, it has shown the feasibility of the technique of underwater neutrino telescopes, which offers important advantages in terms of performance (better angular resolution, better visibility of the Galaxy if built in the Northern Hemisphere). This has paved the way for the next step, KM3NeT, already in construction. Second, the physics harvest of ANTARES is very rich, including many results that show the particular advantages of being in the Mediterranean, as mentioned above. The analyses performed include the search for point-like sources, diffuse fluxes, transient phenomena, dark matter, etc. In this talk we will review this long list of achievements.
\end{abstract}

\section{Introduction}

Neutrino astronomy is part of the new era in our way to observe de Universe. During many years, photons and cosmic rays have been our only probes for that task, but this has changed. The first milestones in this change were the discovery of high energy cosmic neutrinos by IceCube [1,2] and the discovery of gravitational waves by LIGO experiment [3]. More recently, multi-messenger astronomy really started with the observation of a correlation of gravitational waves and electromagnetic signals [4] and of high energy cosmic neutrinos and electromagnetic signals [5]. The importance of these discoveries comes from the fact that gamma rays and cosmic rays have intrinsic limitations that constrain our capability to observe the sky with them. Gamma rays and cosmic rays interact with radiation and matter. Moreover, cosmic rays, being charged particles, are bent by magnetic fields in the Universe, so directional information is smeared. Neutrinos, however, travel unaffected by magnetic fields and only interact weakly, so they are very useful messengers to study regions very far or dense regions of the Universe.

Cosmic neutrinos are expected to be produced in the interaction of cosmic protons and neutrons at their sources. The measurement of the flux very high energy cosmic rays allowed to set the scale of the required neutrino telescopes. This size is of the order of about one cubic kilometre, which is the size of IceCube, in the South Pole.

\footnotetext{
${ }^{\text {a }}$ Corresponding author: zornoza@ific.uv.es
} 
In the quest to access the neutrino window, the two neutrino telescope projects in the Mediterranean Sea, ANTARES and KM3NeT, are also key players, as it will be discussed in the following. Here we will focus on the results already obtained by ANTARES. An overview of the status and prospects of its successor, $\mathrm{KM} 3 \mathrm{NeT}$ can be found in the corresponding proceedings of this conference.

\section{The ANTARES detector}

The ANTARES (Astronomy with a Neutrino Telescope and Abyss environmental Research) telescope [6] is installed off the French coast, at about $40 \mathrm{~km}$ from Toulon, at a depth of 2500 meters. It was completed in 2008 (by 2007 five lines were already in operation and taking data). It consists of 12 lines anchored at the sea bed, with a length of $450 \mathrm{~m}$ and separated by about $70 \mathrm{~m}$ (see Fig. 1). These lines hold a three-dimensional array of 885 photomultipliers (PMTs) to detect the Cherenkov light induced by relativistic leptons produced in interactions of high energy neutrinos with nucleons in the surrounding water and rock. The PMTs and their electronics are hold in pressure resistant spheres called Optical Modules (OMs). These OMs are grouped in triplets for a better rejection of the optical background and point 45 degrees downwards. Additional electronics are housed in titanium cylinders called Local Control Modules (LCMs). The total instrumented volume is about $0.05 \mathrm{~km}^{3}$. In addition to the OMs, the lines are also equipped with optical beacons, tiltmeters, compasses and hydrophones for calibration and positioning purposes. These devices provide a precision of $\sim 1 \mathrm{~ns}$ in time [7] and $\sim 10 \mathrm{~cm}$ in position [8].
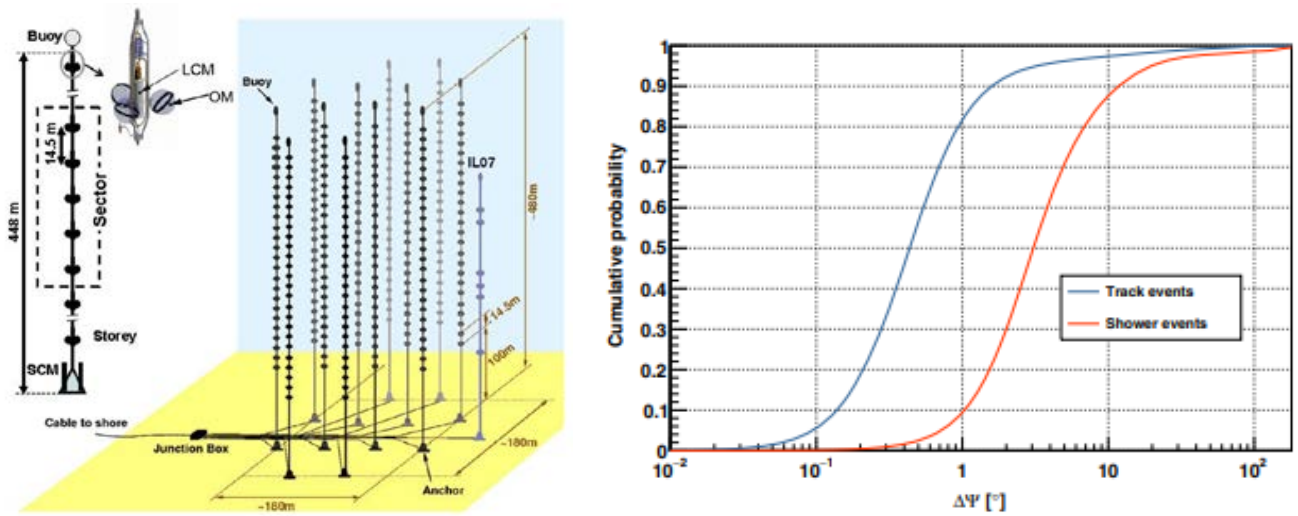

Figure 1. Left : Layout of the ANTARES detector. Right : Angular resolution for the sample of events for pointlike source searches.

There are two main event topologies in neutrino telescopes. Muons, produced in muon-neutrino charged-current interactions, are very penetrating, so they produce long (hundreds of meters) tracks of Cherenkov light. Given the long lever-arm of these events, their direction can be reconstructed with very good accuracy (about $0.4^{\circ}$ of median) (see Fig. 1). On the other hand, neutral-current interactions of any flavour and charged-current interactions of electron and tau neutrinos are seen as bright spheres of light (cascade events), for which it is harder to reconstruct well the direction. Still, given the excellent optical properties of deep sea water, the angular resolution is as good as 4 degrees.

The physical background in neutrino telescopes when searching for astrophysical sources comes from atmospheric muons and atmospheric neutrinos, both produced by cosmic rays interacting in the atmosphere (in a very similar way as the neutrino signal is produced at the sources). In order to reject the (dominant) component of atmospheric muons, the usual strategy is to select only events which are reconstructed upwards, since atmospheric muons cannot traverse the Earth. However, given the large rate of atmospheric muons, it is also necessary to set selection cuts to ensure to avoid that 
misreconstructed events from downgoing atmospheric muons contaminate the neutrino sample. Concerning the background from atmospheric neutrinos, different strategies can be followed, depending on the analysis. Among them, we can mention the fact that the spectral index for this background is softer that what is expected for cosmic signals, so a cut in the energy improves the purity of the sample.

\section{Astronomy}

Best candidates to produce high energy neutrinos detectable by neutrino telescopes are some of the most violent process in the Universe, where nucleons are accelerated at energies and produce charged pions after their interactions with ambient radiation or matter. These pions will decay and produce neutrinos and muons, which in turn also decay and produce more neutrinos. In addition, gamma rays are also expected to be produced in these interactions, coming from the production of neutral pions. Astrophysical sources where these processes should happen include supernova remnants, microquasars and soft-gamma repeaters (among the Galactic sources) and gamma-ray bursts, active galactic nuclei and star burst galaxies (among the extragalactic sources).

\subsection{Diffuse fluxes}

The signal of cosmic neutrinos detected by IceCube seems to correspond to a diffuse, extragalactic flux. A (subdominant) Galactic component is also possible, as maybe hinted by the differences in the best fits for the different searches performed by IceCube.

Searches for diffuse fluxes take advantage of a larger signal, since all the flux in the visible sky is integrated. The price to pay is a larger background, which can be reduced by a cut in the energy of the events. In a recent analysis [9], ANTARES has searched in a data sample of nine years, including tracks and showers and found a small (non-significant) excess compatible with the IceCube signal. This analysis excludes the absence of a cosmic flux at a level of $1.6 \sigma$.

Another example of search for a diffuse flux is the analysis of the region of the so-called Fermi Bubbles [10] (see Fig. 2). The gamma rays detected by the Fermi experiment could be explained by hadronic mechanisms which would also produce high energy neutrinos.

Finally, a search in the Galactic plane has also been recently published [11]. No significant excess has been found, but constraints to close to the predictions of the KRA model [12] have been set.

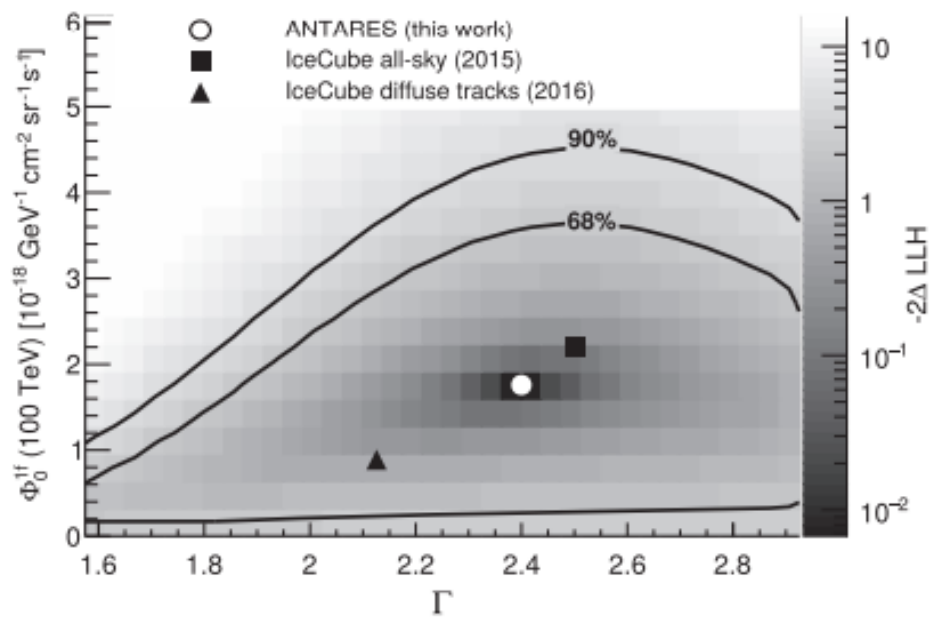

Figure 2. 2D log-likelihood scan of the diffuse cosmic flux normalization and spectral index. Black lines indicate $90 \%$ and $68 \%$ confidence levels. 


\subsection{Point-like sources}

The search for point-like sources is one of the key analyses in ANTARES, since it has a clear competitive advantage to observe the Southern sky, where most of the Galaxy, including the Galactic Centre, is best observed by neutrino telescopes, since the Earth can be used as an efficient filter for atmospheric muon rejection as previously explained. In a recent analysis [13], ANTARES has carried out several types of searches for point-like sources using data from 2007 to 2015, including 1) fullsky, 2) search in a list of locations of sources candidates for emitting high-energy neutrinos, 3) a region of about $20^{\circ}$ around the Galactic Centre, and 4) a search around Sagittarius A*. Figure 3 shows the sensitivities and limits obtained in the list of candidates sources. These recent searches included, for the first time, the contribution from cascade events. Although the angular resolution of these events is not as good as for tracks, in water it is still good enough to be used for these kind of searches. No excess has been found in these searches (see Fig. 3).
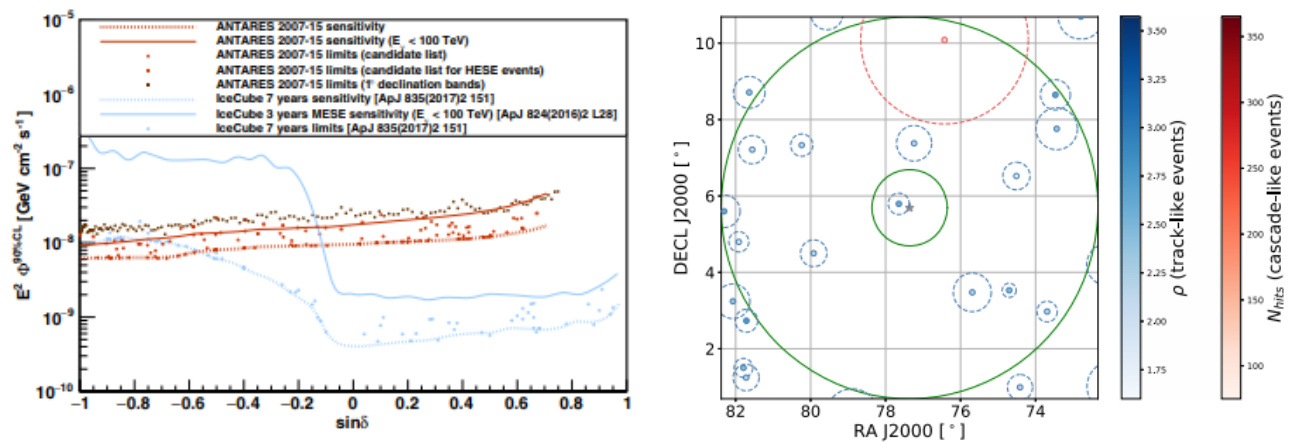

Figure 3. Left: Limits in the cosmic neutrino flux as a function of the declination of the source. Sensitivities are also shown assuming a $\mathrm{E}^{-2}$ spectrum with no cut off (solid line) and a cut off in the neutrino energy at $100 \mathrm{TeV}$.

Recents reults from IceCube are also shown. Right: Events around the most significant cluster in the all-sky search.

\subsection{Multi-messenger}

As mentioned before, the recent years have witnessed the birth of a multi-messenger astronomy, in which all the cosmic messengers are participating. ANTARES has participated in several of these searches, looking for correlations of its data with gravitational wave events, high energy neutrinos and electromagnetic signals. As an example of this effort, Figure 4 shows the results of the search for a correlation of events in the ANTARES data with the gravitational wave event GW170104. No neutrino candidates were found in a window of $\pm 500 \mathrm{~s}$ around the event and limits on the neutrino fluence were set [14].
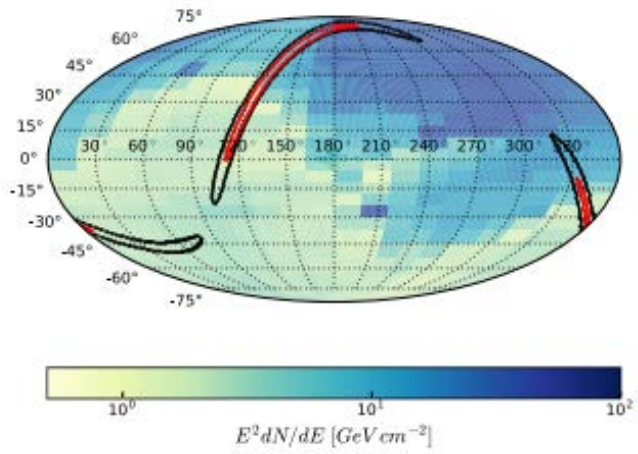

Figure 4. All-sky upper limits on the neutrino fluence from GW170104 assuming a $\mathrm{E}^{-2}$ spectrum emitted at the source. Countrous for $68 \%$ and $90 \% \mathrm{CL}$ are also shown. The skymap is shown in equatorial coordinates. 


\section{Particle physics}

Particle physics is also a main domain among the scientific goals of ANTARES. This include dark matter, neutrino properties and magnetic monopoles.

\subsection{Dark matter}

Dark matter is one of the most pressing question in fundamental physics today. Since we do not know what dark matter is made of, it is impossible to pin-point the best strategy to discover it. Each method has its own advantages. Neutrino telescopes offer a complementary way to explore this unknown side of Nature. Among several interesting sources, the Sun and the Galactic Centre are the best candidates to look for. In the case of the Sun, we expect that dark matter particles that encountered by the Sun as it travels around the centre of the Galaxy would scatter off the nucleons in the Sun, lose energy and eventually become gravitationally trapped. These dark matter particles are expected to self-annihilate to several products which would include high energy neutrinos as secondaries. A potential signal of high energy neutrinos in the direction of the Sun would be a smoking gun of dark matter, since, contrary to most of other indirect searches, the astrophysical backgrounds are known and low. If we assume that, as it is expected, there is an equilibrium between capture and self-annihilation, we can set limits on the scattering cross section of DM-nucleon [15].

For the Galactic Centre, limits are set on the thermally averaged annihilation cross-section of dark matter particles. For masses above $30 \mathrm{TeV}$, ANTARES has set the best limits worldwide [16] (see Fig. 5).

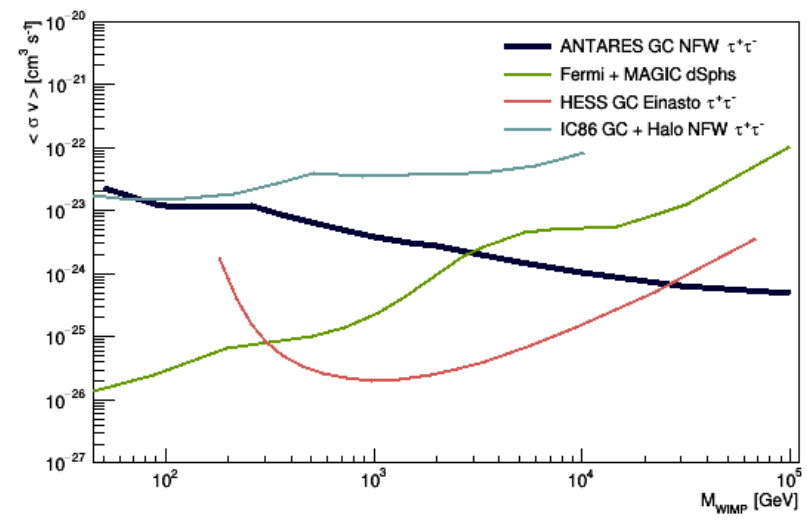

Figure 5. ANTARES limits on the thermally averaged annihilation cross-section as a function of the dark matter particle mass. Results of other experiments are also shown for comparison.

\subsection{Oscillations}

ANTARES has also shown capability to observe neutrino oscillations. As a matter of fact, the first hints of neutrino oscillations set by neutrino telescopes were obtained by this experiment [17]. In a more recent analysis, using ten years of data, a measurement of $\theta_{23}$ and $\Delta \mathrm{m}_{32}{ }^{2}$ has been made, consistent with the world best fit values. Moreover, these data have also been used to constrain $3+1$ models of sterile neutrinos. These results are shown in Fig. 6 [18]. 

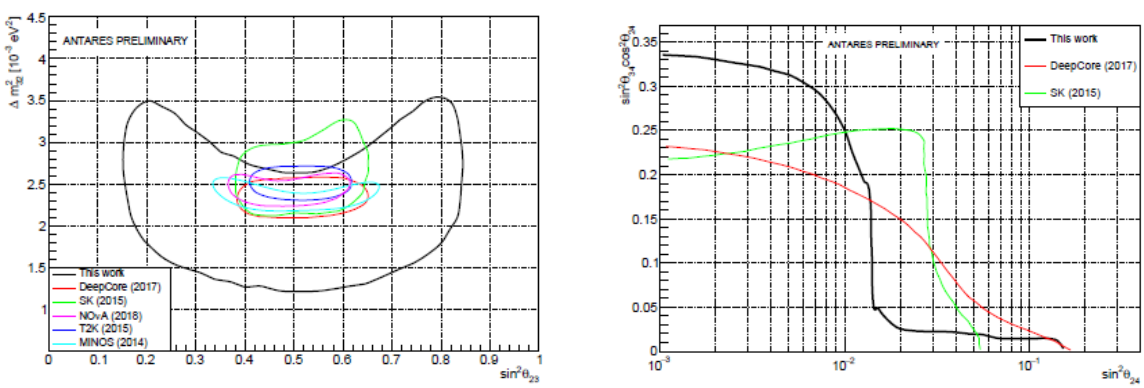

Figure 6. Left: Contour at $90 \% \mathrm{CL}$ in the plane of $\theta_{23}$ and $\Delta \mathrm{m}_{32}{ }^{2}$ using ANTARES data from 2007 to 2016. Right: Constraints on $3+1$ models of sterile neutrinos in the parameter space of $\left|U_{\mu 4}\right|^{2}=\sin ^{2} \theta_{24}$ and $\left|U_{\tau 4}\right|^{2}=\sin ^{2}$ $\theta_{34} \cos ^{2} \theta_{24}$.

\section{Conclusion}

ANTARES started taking data in 2007. Since then, a very rich harvest of physics analyses has been produced, both for astronomy and fundamental particle physics. These results include searches for diffuse fluxes, point-like sources, multi-messenger, dark matter and neutrino properties. This effort has shown that underwater neutrino telescopes are feasible and that they have the capability to profit from the advantages of neutrino astronomy in the Mediterranean: great angular resolution and very good visibility of our Galaxy.

The successful experience of ANTARES is a solid base for the next great leap, the KM3NeT detector, the construction of which has already started.

\section{References}

1. IceCube Collaboration. Science. 342 (2013) 1242856.

2. M.G. Aartsen, M. Ackermann, J. Adams, J.A. Aguilar, M. Ahlers, M. Ahrens, D. Altmann, T. Anderson, C. Arguelles, T.C. Arlen. Phys Rev Lett. 113 (2014) 101101.

3. B. P. Abbott et al. (LIGO Scientific Collaboration and Virgo Collaboration), Phys. Rev. Lett. 116, 061102

4. B. P. Abbott et al., ApJL 848 L12 (2017)

5. IceCube and other colls., Science 12 Jul 2018

6. M. Ageron et al., Nucl. Instrum. Meth. A 656 (2011) 11-38

7. J.A. Aguilar et al., Astropart. Phys. 34 (2011) 539-549,

8. S. Adrian-Martinez et al., JINST 7 (2012) T08002

9. A. Albert et al., ApJL 853, L7 (2018)

10. S. Adrián-Martínez et al., Eur. Phys. J. C (2014) 74:2701

11. Adrián-Martínez et al., Physics Letters B 760 (2016) 143

12. D. Gaggero, D. Grasso, A. Marinelli, A. Urbano, M., Valli, ApJL, 815:L25 (2015)

13. A. Albert et al., Phys. Rev. D 96, 082001 (2017)

14. A. Albert et al., ApJL 850 L35 (2017)

15. Adrián-Martínez et al., Physics Letters B, Volume 759, 10

16. A. Albert et al., Phys. Let. B 769 (2017) 249,

17. S. Adrian-Martinez et al., Phys. Lett. B 714 (2012) 224-230

18. Salvadori, I. (2018), Zenodo. http://doi.org/10.5281/zenodo.1300869 